\title{
An automated system for mapping spatial movement patterns in behavioral research
}

\author{
PAUL NISWANDER and WILLIAM LOADMAN \\ Ohio State University, Columbus, Ohio \\ KAYE RASNAKE \\ Denison University, Granville, Ohio \\ and \\ JOHN WELLS \\ Bikron Corporation, Columbus, Ohio
}

\begin{abstract}
A computerized positioning system was developed and used to map gross body movement of children around a room in response to high-intensity sound stimulation. Details of the data collection system are discussed. The system has potential for use in a variety of research projects when it is necessary to identify position coordinates and analyze changes in spatial location for subjects within a test room environment.
\end{abstract}

In this paper we describe a computer-based positioning system that we designed for collecting and archiving gross body movement data from children. Pressuresensitive tiles were installed in a grid pattern on the floor of a behavioral analysis lab and interfaced with a computer. The movement of a child around the lab room was determined by programming the computer to sample the tiles at regular intervals to identify depressed tiles. Records of depressed tiles were stored in text files on the computer hard disk. From these data, the position of the child at any instant in time could be determined, and derivations of position such as velocity (speed and direction) and acceleration could be calculated. Additionally, maps of cumulative movement patterns were provided by means of an animation program that produced graphical displays of the movement. These maps were stored on videotape for later analysis and could be used to examine movement patterns occurring within a selected time interval.

In addition to the data collected by the grid system, supplemental behavioral responses were captured by video cameras mounted in the behavioral analysis lab room. The camera outputs were multiplexed with the real-time grid display for viewing on a monitor. In addition, the outputs of the cameras were multiplexed and recorded on videotape along with the movement maps from the computer. If analysis of movement was the sole consideration, however, the supplemental cameras could be omitted.

This work was supported by SBIR Grant N41-HD-2911 from NIH, entitled "Sound in Prevention of Child Electrical Injury." Address reprint requests to P. S. Niswander, The Nisonger Center, Ohio State University, 1581 Dodd Drive, Columbus, OH 43210 (614-292-9605).
Our system required custom construction of the pressure-activated tiles because prefabricated tiles that met our cost, size, and sensitivity requirements were not available. Although component parts for construction of tiles similar to those we used are currently available, the recent introduction of pressure-sensitive resistors provides a possible source of ready-made sensors. These are described briefly in the Appendix. Computer programs had to be written to carry out the necessary sampling functions from the tiles, but suitable software is now available commercially.

Our system originally was designed and constructed for use in a study measuring the aversive effects of highintensity sounds on children. However, the system would be ideally suited for a variety of research applications requiring measurement of gross body movement.

For example, body movement measurements have been of particular importance in the area of behavior disorders in children and adolescents. The need to quantify activity levels and determine the effects of medications has led to the development of a variety of mechanical and/or electronic measurement techniques. A discussion of methodological considerations of various approaches is presented in Aman and White (1986), and an overview of current measurement techniques can be found in Aman (1993). The interested reader is referred to these publications for further information.

There are other areas, such as studies involving sleep disorders and somnambulism, for which automatic recording of movement would be desirable. In general, this system would lend itself well to any investigation in which movement of an individual was of interest and could be confined within a single test room. 
An extremely valuable aspect of our system is the fact that data are fed directly into a computer and hence are available for immediate analysis. Therefore, modifications in experimental variables in order to achieve specified criterion outcomes can be made quickly and easily. For studies that cannot accommodate long delays in analysis turnaround, our system has distinct advantages.

In the following text we describe the various components of our positioning system. We also describe construction of the tiles in the event the requirements of individual investigators are not met with commercial tiles. Commercial sources of various components of the system are provided in the Appendix.

\section{SYSTEM DESCRIPTION}

\section{General Design Considerations}

Initially, we investigated several systems for collecting our movement data. Several considerations guided our selection process. First, of course, we required a system that provided sufficient resolution of position and movement so that the effect of the experimental variables could be studied adequately. We needed to know exactly where the child was in the room, in what direction he/she was headed, and at what speed. Further, we wanted to have a system that would allow us to generate movement maps so that patterns of movement over specified time intervals could be studied.

Our second requirement was only slightly less important than our first. We needed to have a system that would allow us rapid turnaround in data analysis. We would be engaged in a series of experiments in which the design of each depended on the results of those preceding. We would be engaged in tight deadlines and so did not have the luxury of long down periods for data analysis. We did not want to store data on a medium that required us to go back and transcribe for computer entry, because we would not have the time to do this. Ideally, we wished to identify a system that would allow us to input our data directly to a computer for reduction and analysis.

Finally, we did have cost and reliability constraints. We wished to have a system that was as economical as possible to acquire and operate, relatively simple to install and maintain, and reliable in operation.

One approach we considered involved ceilingmounted video cameras with a grid laid out on the floor so that direction and speed of movement could be assessed from videotapes of the subject moving around the room. However, because of the relatively low ceiling in the experimental room, six to eight cameras would have been required to provide full coverage of the room and their outputs multiplexed for full-room viewing. A greater limitation, from our perspective, was the fact that this type of system would involve initial storage of data on videotape, frame-by-frame videotape analysis and data coding, and, finally, entry of the data into a computer for analysis. As noted previously, we needed to avoid any system that involved lengthy analysis procedures or that could not input our data directly to a com- puter. In addition, it appeared that the generation of movement-pattern maps would require considerable effort. From a technical point of view, videotaping appeared to be perhaps the most simple system. From the data storage and analysis standpoint, however, it seemed to be perhaps the most complicated.

Other systems currently used for providing position information include sonar and infrared devices. However, each appeared to have its own limitations with respect to the requirements of the proposed study.

Sonar-based systems are frequently used in cameras for focusing purposes and also in some intrusion alarm systems. On the basis of the former application, it would appear that a sonar system would be suitable for determining the distance of an object from a sonar source. A single sonar source could scan the room and provide position information. However, because of the location of the study in a rather small room with hard-surfaced walls, we discovered that problems would arise because of sonar reflections or echoes from the wall. Distance resolution of the location of a subject would be compromised at best or ambiguous at worst. Moreover, our experimental design called for the presence in the test room of both a parent and several large toy objects in addition to the child. We discovered that sonar shadows would exist behind these objects and the child's location could not be determined if he/she entered one of these shadow zones. With the various objects present, a considerable portion of the room would be within such a shadow zone. It appeared, therefore, that a sonar-based system would not meet our needs.

Another system used for detecting location and movement is based on infrared measurements. Most infrared applications require only that the presence of an object within a protected area be indicated, however, with position information not required. Nevertheless, some infrared applications do provide position information. A good example of such a system is the touch screen for registering finger location on the faceplate of a computer monitor screen. However, setting up a touch screen on a computer monitor is quite different from constructing an infrared detection system in a room. In order to achieve a location resolution of about 6 in., which was what we had specified for our study, a series of infrared beams separated by no more than $12 \mathrm{in}$. would have to be directed across the room. Moreover, distance of placement of these beams above the floor would be critical to ensure that the child did not step between the beams. Each beam would require an infrared source, a detector, and an amplifier, an arrangement that would entail considerable expense. Of perhaps more concern was the fact that shadow problems would also plague this system. The other objects in the test room would interrupt the infrared beams and thereby cause shadows along the entire length of the interrupted beams so that no measurements could be conducted there. As with the sonar system, an infrared system would find a considerable portion of the room blacked out. In addition, ambient light levels would need to be carefully controlled to ensure that de- 
tectors were not accidently triggered. It appeared, therefore, that an infrared system would not meet our needs.

The remaining approach, which seemed feasible for gathering the required data, involved the use of pressureactivated tiles on the floor of the test room. The outputs of such tiles, which are basically switch openings and closings, are admirably suited for interfacing with a computer. Stationary objects located in the room could be ignored either by disconnecting tiles activated by those objects or by software commands instructing the computer to ignore input from the particular tiles upon which the objects had been placed. Even though a large number of tiles would be required to cover the entire floor, it appeared that the cost of the tiles would not be prohibitive and, except for wiring supplies, would be the major expense associated with this particular approach.

The primary drawback for the pressure-sensitive-tile system appeared to be the necessity for covering the entire floor surface of the test room with the tiles and the subsequent wiring required to provide input to the computer, since each tile would need to interface with the computer. This would require some rather tedious hookup work, but would not be particularly complicated. In addition, no subsequent alignment or periodic calibration of the system would be required.

On the basis of these various considerations, then, it appeared that the pressure-sensitive-tile approach was the only system that would meet our needs. We understand that a different system might meet the needs of other experimenters who have different data collection requirements. All things considered, however, we believe that a mapping system based on pressure-activated tiles is an excellent choice because it is very versatile, quite simple in operation, and allows for rapid data reduction and analysis.

\section{Construction of Pressure-Sensitive Tiles}

When we originally constructed our system, we investigated several commercial pressure pads, but found none of them to be sufficiently sensitive for use with children as young as those to be included in our study. Our own pilot studies indicated that our youngest children, who fell within the age range from 18 to 24 months, were in many cases too light to activate the commercial pressure pads. In addition, if they did activate a tile by standing on it, they tended to "disappear" and "reappear" as they walked, since their weight at various times was distributed between several tiles and was therefore insufficient to activate any single one of the contacted tiles. Therefore, we constructed our own tiles in the manner to be described.

Figure 1 shows general details of the pressure-sensitive tiles. Touch strip elements, which are basically on/off switches that can be activated by pressures as little as approximately $8 \mathrm{oz}$, were purchased in $6-\mathrm{ft}$ lengths and cut into 8.5 -in. lengths. Both ends of the cut pieces were hermetically sealed with a silicon sealer. Three of these lengths were attached in parallel with two-sided adhesive tape to a 9 -in. square piece of $1 / 8$ in. thick tempered

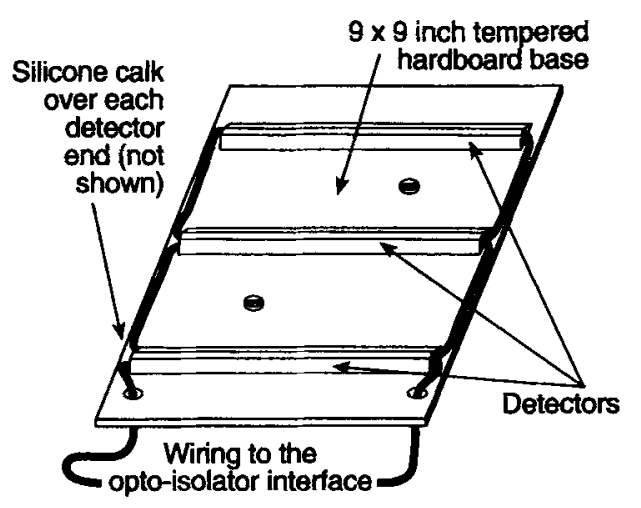

Figure 1. Sketch of a pressure-sensitive tile showing general construction details.

hardboard base. The three touch strips on each tile were wired in parallel so that the entire tile was activated by pressure on at least one of the touch strip elements.

Our use of 9-in. tiles was somewhat arbitrary. In general, position resolution of the system will be one half the size of the tile used. For our system, which incorporated 9 -in. tiles, position resolution was approximately $4.5 \mathrm{in}$. We determined this to be adequate for our requirements, although other investigations might need either greater or lesser resolution. In such cases, both the size of the tiles and the number of elements per tile could be modified.

\section{Installation of Pressure-Sensitive Tiles}

Our tiles were attached with screws to the floor of our test room, which we called the "grid room." Figure 2 is a sketch of the grid room with the tiles on the floor and the video cameras mounted on the walls. Perhaps the greatest challenge of the installation project was the large number of wire leads that needed to be attached from the tiles to the computer. Our own installation required 240 tiles. Since each tile had two lead pairs that required connection to the computer via a separate input channel, a total of 480 wiring connections to the computer were required to complete the installation.

Alternative methods of connecting the tiles to the computer to simplify the wiring task did not appear to be practical. For example, an $x-y$ matrix system could have been employed using column and row wires with each tile connected to a column wire and to a row wire. The computer then would identify a depressed tile based on the $x-y$ coordinates of that tile. The first major problem with this approach is that it does not allow for easy identification of multiple tile depressions. A further problem in our system was that the interpreted software program did not run sufficiently fast on our computer so that coordinates on depressed tiles could be determined from a matrix system and movement data could be gathered at the same time.

Our installation site allowed us access to the gridroom floor from underneath, and this facilitated tile hook-up. If such access is not possible, as would be the 


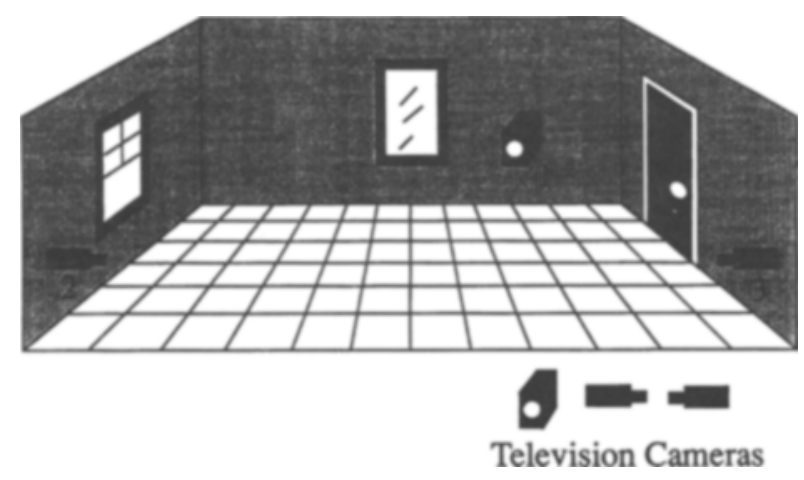

Figure 2. Drawing of grid room showing pressure-sensitive tiles on the floor and approximate locations of the three video cameras.

case with a poured concrete floor, a different wiring configuration would be required. We found that running wires between the tiles was not feasible due to the sheer number of wires involved. If access is not possible from underneath the floor, construction of a wood subfloor should be considered. This would allow wire runs to be made underneath the floor and would greatly simplify the electrical hook-up of the system.

The tiles we constructed were slightly bumpy, owing to the thickness of the touch strip elements mounted on the rigid backing material. Originally, we intended to place carpet over the tiles to smooth the surface. However, the stiffness of the carpet backing tended to distribute pressure over several tiles. In some cases, this resulted in false activation of the tiles surrounding the actual location of the child. In other cases, the child's weight was distributed over a sufficiently wide area so that no touch strips were activated at all. We experimented with several materials and found that a light cloth about the weight of bed sheeting could be stapled over the surface of the tiles. Although the resulting surface was slightly bumpy, none of our young subjects seemed to take notice of this situation and mobility did not appear to be inhibited.

\section{Computer System and Associated Components}

Figure 3 is a block diagram summarizing the major components of the recording system. The tiles were connected to a Macintosh II computer through a pair of 256 channel I/O optical isolator boards. These optical interfaces isolated the $1.5-\mathrm{V}$ signals passing through the touch strips from the computer and also prevented transmission of large static electricity spikes to the computer input. Connections between the tiles and the I/O optical isolator boards were made by means of screw terminal blocks using twisted-pair cable from the tiles.

A custom computer program was written to provide position information from the tiles, although commercial programs that provide the same information are now available. Our program scanned the tile inputs twice each second and determined which tiles were activated. Each tile was assigned a unique identification number, and the identification codes of activated tiles at each sample time were saved as text files on a hard disk. The identification codes contained sequence-of-activation information and allowed for the possibility of conducting subsequent time series types of analyses on the data.

If a child happened to be standing in such a way that several tiles were activated, the computer calculated the "center of presence" from the pattern of activated tiles and stored this location. This was done by assigning the location of the child to the tile nearest the geometric center of the activation pattern. Occasionally, a child would attempt to climb onto the lap of the parent, but this was not a serious problem since we had requested prior to the experiment that the parent not allow this during the trial periods.

In our research project, we had objects in the grid room in addition to the child. We seated a parent approximately in the center of the room, and several large toys were situated at various places in the room. Tiles that would have been activated by these objects were disconnected from the computer at the optical isolator inputs so that their activation was not recorded by the computer. However, our computer program also allowed any tiles to be "locked-out" so that their activation was not included in the analysis. Occasionally, tiles would stick in the activated position owing to humidity and malfunction of the touch strips. A computer smoothing paradigm was used to eliminate "outlier" activated tiles from the analysis. This routine functioned by eliminating from analysis any tiles that were depressed continually during the entire trial period.

A HyperCard animation program provided a display of the activated tiles on the computer monitor. The grid arrangement of the room appeared on the monitor screen

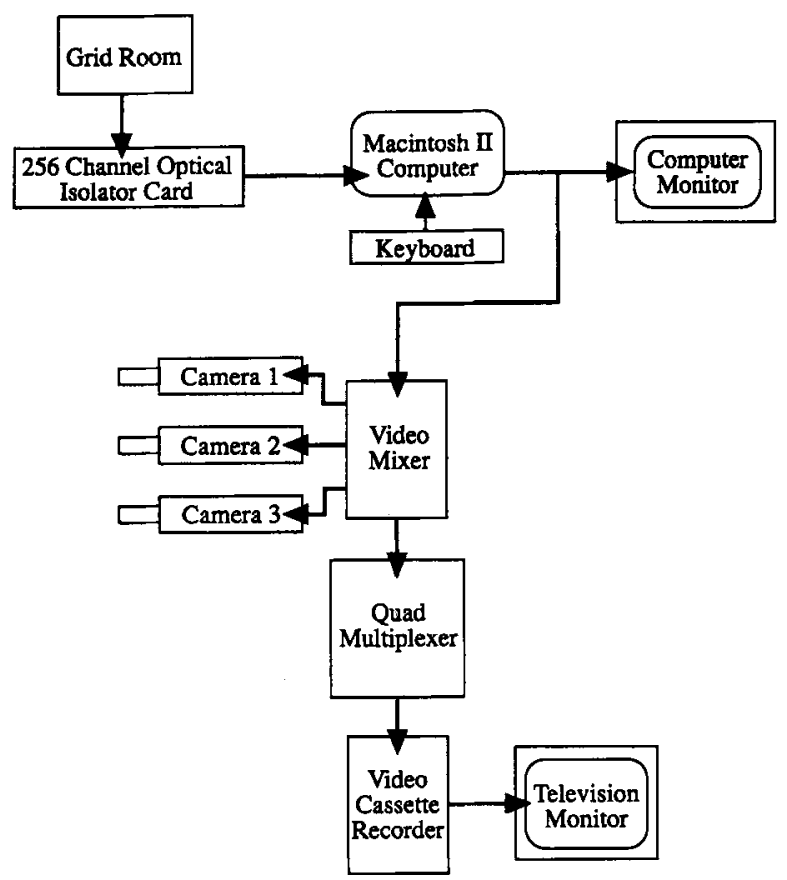

Figure 3. Block diagram of various components in the data collection system. 


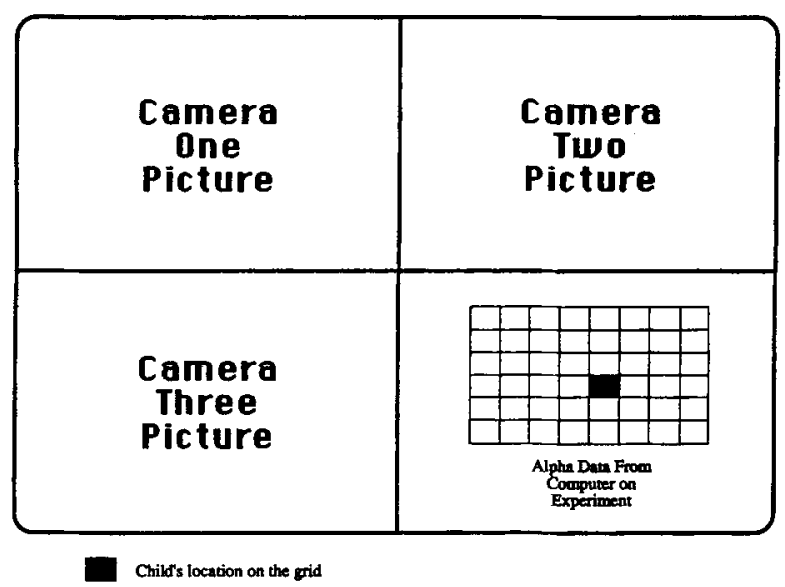

Figure 4. Multiplexed video signal combining the video outputs of the three video cameras and the positioning data from the computer, as displayed by the television monitor and recorded on videocassette tape.

with the activated tiles showing as black squares. Position information was updated on the monitor display every second (i.e., every other sampling scan). It was very easy to follow a child around the test room on the monitor display by noting the progression of darkened squares on the computer monitor. These movement maps were recorded on videotape for subsequent analysis.

Our project required us to have a record of other behavioral responses of the children in addition to the movement information. For example, when presented with the sounds, some of the children froze and covered their ears with their hands. We collected this additional behavioral data by placing three small video cameras around the grid room so that a frontal view of the child's face and a record of other body movement was available regardless of the child's position in the room. The outputs of the three video cameras were combined with the animated grid display from the computer via a quad multiplexer and displayed on a TV monitor. The quad multiplexer we used was designed for security system use and did not require input sources to be gen-lockable. The output of the quad multiplexer, besides being connected to the TV monitor, was also fed to a videocassette recorder, where it was recorded. Playback of the videotape allowed us to observe simultaneously the pattern of tile activation and the additional behavioral responses captured by the three video cameras. Figure 4 provides an illustration of the multiplexed display of the video cameras and grid system on the TV monitor.

\section{DATA ANALYSIS}

Statistical analyses were completed on data in the stored text files acquired during approximately 30 -sec trial intervals. It was a simple matter to determine the position of the child at any point in time within a sampled time interval. Total linear distance moved over any predetermined time interval could be calculated, and relative measures of stationary time versus moving time were easily determined. In addition, velocity (speed and direction) of the child between any two location points could be calculated. Acceleration could be calculated from velocity changes between any two location and/or

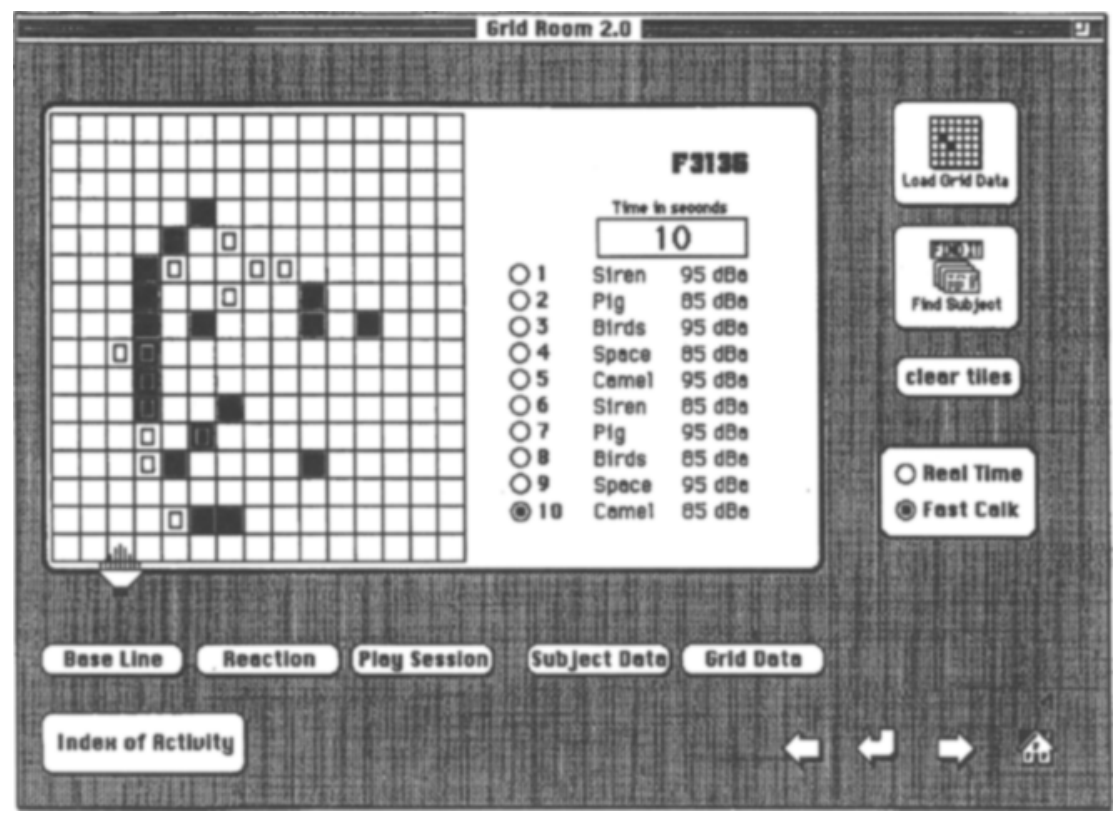

Figure 5. Screen print of the movement pattern of a subject generated by the HyperCard animation program. Additional information relevant to the research is included on the screen. The unfilled squares denote prestimulus location, and the filled squares denote poststimulus location. 
time points. All of the above variables were subjected to various statistical analyses in order to address the original research questions.

A more qualitative analysis of movement was provided by the supplemental graphical mapping of the child's movement around the grid room from the HyperCard animations that were recorded on videotape. Patterns of movement could be reconstructed and subjected to analysis. Figure 5 is a screen print of the movement pattern of a child over a several-second interval that was generated by the HyperCard animation program. Summarized in this figure is the child approaching the loudspeaker (unfilled squares) and then retreating (filled squares) upon presentation of the sound. Activated tiles away from the direct path of the child are caused by toy objects and/or the parent in the test room; we locked out these extraneous activated tiles when data were analyzed. The general orientation of the room and the child's location in it are preserved on the monitor display. For example, the loudspeaker symbol located on the grid represents the approximate location of the activated loudspeaker in the test room. As noted previously, the videotapes that recorded these movement patterns also included the additional behavioral responses from the three video cameras.

\section{FINAL COMMENTS}

We found that our system met our requirements for determining gross body movement. In addition, it allowed us to capture other behavioral responses that were important for addressing our research questions.

An extremely useful aspect of our system was that it was completely automated. Although we did not do so, it would be possible to collect data while the system is unattended. Data could be collected over periods as short as seconds or as long as hours with only a few minor changes in the computer program controlling the data collection. Behavioral effects of a particular medication of interest might be determined during a severalhour experimental session by sampling movement during, say, a 20 -sec interval every $10 \mathrm{~min}$. For sleep studies, the system could be set up to gather data for an entire night. The text files used to store positioning information directly from the grid system are quite efficient and store large amounts of information in relatively small files. Therefore, large hard disks are not required. With the data storage capabilities of currently available microcomputer systems, the task of collecting, storing, and retrieving large amounts of data is accomplished easily.

\section{REFERENCES}

AMAN, M. G. (1993). Monitoring and measuring drug effects: II. Behavioral, emotional, and cognitive effects. In J. S. Werry \& M. G. Aman (Eds.), Practitioner's guide to psychoactive drugs for children and adolescents (pp. 99-159). New York: Plenum Medical.
AMAN, M. G., \& WhITE, A. J. (1986). Measures of drug change in mental retardation. In K. G. Gadow (Ed.), Advances in learning and behavioral disabilities (pp. 157-202). Greenwich, CT: JAI Press.

\section{APPENDIX}

Software and data acquisition boards can be purchased from a variety of vendors and our list of potential sources is not meant to be inclusive. Several sources we are aware of are as follows:

Kaye Instruments, Inc.

15 DeAngelo Drive

Bedford, MA 01730

Microstar Laboratories

2265116 th Avenue N.E.

Bellevue, WA 98004

National Instruments

6504 Bridge Point Parkway

Austin, TX 78730-5039

Strawberry Tree

160 South Wolfe Road

Sunnyvale, CA 94086

A copy of the software that we developed for data collection is available without charge. Note that this software was developed to run on a Macintosh II computer under MicroSoft BASIC and was written to support the Strawberry Card. A copy of the software may be obtained by writing to:
John Wells
Bikron Corporation
1208 E. Hudson Street
Columbus, $\mathrm{OH} 43211$

Pressure-sensitive pads have been widely used for detection and door-opening systems, but their use has been largely supplanted by systems based on infrared and sonar technology. As a result, pressure pads are becoming increasingly difficult to purchase. The company that supplied our pressure-sensitive strips apparently is now out of business. However, pressure strips and several styles of pressure pads of various sizes and shapes can be obtained from the following source:

\section{Tapeswitch Corporation of America 100 Schmitt Boulevard Farmington, NY 11735}

A particularly promising pressure-sensitive sensor recently has become available with the introduction of force-sensing resistors. These wafer-like devices are very sensitive, very thin (approximately .050 in. thick), and can be custom made to almost any size. They come with an attached molded plug to simplify hook-up. Information on these devices can be obtained from:

Interlink Electronics

1110 Mark Avenue

Carpinteria, CA 93013-2918

(Manuscript received December 4, 1992; revision accepted for publication January 31,1994 .) 